\title{
Identification of Mutated Peptides in Bladder Cancer From Exomic Sequencing Data Reveals Negative Correlation Between Mutation- Specific Immunoreactivity and Inflammation
}

OPEN ACCESS

Edited by:

Peter Brossart,

University of Bonn, Germany

Reviewed by:

Jing Li,

Second Military Medical University,

China

Anna Pasetto,

Karolinska Institutet (KI), Sweden

*Correspondence:

Ying Wang

ywang@sibs.ac.cn

Haibo Shen

shenhaibo@xinhuamed.com.cn

${ }^{+}$These authors have contributed equally to this work

Specialty section:

This article was submitted to Cancer Immunity and Immunotherapy,

a section of the journal

Frontiers in Immunology

Received: 26 June 2020 Accepted: 02 November 2020 Published: 30 November 2020

Citation:

Wang C, Ding Y, Liu Y, Zhang Q, Xu S, $X i a L$, Duan $H$, Wang $S$, Ji $P$, Huang $W$, Zhao G, Cao Z, Shen $H$ and Wang $Y$ (2020) Identification of Mutated Peptides in Bladder Cancer From Exomic Sequencing Data Reveals

Negative Correlation Between Mutation-Specific Immunoreactivity and Inflammation.

Front. Immunol. 11:576603. doi: 10.3389/fimmu.2020.576603
Chen Wang ${ }^{1 \dagger}$, Yu Ding ${ }^{1+}$, Yuanyong Liu ${ }^{2+}$, Qingchen Zhang ${ }^{3}$, Shiqiang $\mathrm{Xu}^{2}$, Liliang $\mathrm{Xia}^{2}$, Huangqi Duan ${ }^{1}$, Shujun Wang ${ }^{2}$, Ping $\mathrm{Ji}^{2}$, Weiren Huang ${ }^{4}$, Guoping Zhao ${ }^{5}$, Zhiwei Cao ${ }^{3}$, Haibo Shen ${ }^{1 *}$ and Ying Wang ${ }^{2,5 *}$

\footnotetext{
${ }^{1}$ Department of Urology, Xinhua Hospital, Shanghai Jiaotong University School of Medicine, Shanghai, China, ${ }^{2}$ Department of Immunology and Microbiology, Shanghai Institute of Immunology, Shanghai Jiaotong University School of Medicine, Shanghai, China, ${ }^{3}$ Deparment of Gastroenterology, Shanghai Tenth People's Hospital, School of Life Sciences and Technology, Tongji University, Shanghai, China, ${ }^{4}$ State Engineering Laboratory of Medical Key Technologies Application of Synthetic Biology, Shenzhen Second People's Hospital, The First Affiliated Hospital of Shenzhen University, Shenzhen, China, ${ }_{5}^{5}$ Shanghai-MOST Key Laboratory of Health and Disease Genomics, Chinese National Human Genome Center at Shanghai, Shanghai, China
}

Introduction and Objective: Neoantigen-based immunotherapy is one of the breakthroughs in cancer immunotherapy. Benefit from the Cancer Genome Atlas database, we intended to identify mutant peptides with neoantigen property in bladder cancer (BC). Correlations between the immunoreactivity of candidate neoantigens and clinical manifestations were further analyzed.

Methods: HLA-A*02:01 restricted mutant (MT) and wildtype (WT) peptides were predicted by using whole exome sequencing data of 412 BC patients in the TCGA database. Binding affinity to HLA-A2 molecules was determined by using T2 cell-based binding assay. The immunoreactivity to $\mathrm{WT}$ and $\mathrm{MT}$ peptides in $\mathrm{HLA}-\mathrm{A} 2^{+} \mathrm{BC}$ patients was determined by using an ELISPOT assay upon in vitro stimulation with MT and WT peptides individually. Clinical relevance to peptide-specific immunoreactivity was analyzed by Pearson correlation analysis. The disease free survival (DFS) curves were plotted using the Kaplan-Meier method in BC patients with or without mutations and compared using the log-rank test online.

Results: Fifty-seven HLA-A*02:01 restricted WT and MT peptides were selected based on predicted high affinity and expression frequency, among which 12 MT peptides from 12 individual genes exhibited strong affinity to HLA-A2 molecules when compared to WT counterparts. MT peptides induced more peptide-specific IFN $\gamma$ spot forming units (SFUs) than WT counterparts in HLA-A2 ${ }^{+}$BC patients upon in vitro stimulation. They were negatively correlated to the counts of peripheral leukocytes and platelets. Patients with 
higher C-reactive protein level exhibited lower immunoreactivity to MT peptides. Combination of MT peptides from 6 genes, including CDKN1A $A^{G 61 V}, R H O B^{P 75 L}$, $D D B 1^{S 25 L}, A H N A K^{D 4855 Y}, A N P 32 A^{S 56 L}$ and MKI67 ${ }^{H 84 L}$ covered $47.5 \%$ of the patients under investigation. Patients harboring combinational mutations in these genes were associated with a longer DFS according to the cBioportal online analysis.

Conclusion: Twelve HLA-A*02:01 restricted MT peptides have been identified exhibiting higher binding affinity to HLA-A2 molecules and stronger immunoreactivity than WT counterparts in BC patients. Combination of MT peptides from six genes might be potential as neoantigen candidates in cancer immunotherapy against BC in the future. Inflammatory modulation is inclined to be a strategy to enhance the efficacy of neoantigenbased immunotherapy.

Keywords: bladder cancer, the Cancer Genome Atlas database, mutated peptides, immunoreactivity, inflammation, neoantigen

\section{INTRODUCTION}

Bladder cancer (BC) originates from transitional cells of bladder urothelium. It is the sixth most common cancer in Europe and the United States $(1,2)$. In China it is estimated with 80,500 new cases and 32,900 deaths of BC in 2015 (3). The vast majority of patients are diagnosed as non-muscular invasive bladder cancer (NMIBC) and receive transurethral bladder tumor resection. At present, the 5-year recurrence rate of NMIBC is $31-78 \%$. Up to $45 \%$ of the patients at the first follow-up cystoscopy have suffered recurrence, and $6-17 \%$ of them progress into muscle invasive bladder cancer in the long-term follow-up (4). The etiology and pathophysiology of BC are not well addressed yet. New strategies to improve the clinical diagnosis and the treatment of $\mathrm{BC}$ are thus still challenging.

Besides the surgery and chemotherapy, intravesical administration of Bacille Calmette-Guerin (BCG) is one of the most successful examples of immunotherapy through inducing regional and systemic anti-tumor immunity $(5,6)$. In fact, cancer immunotherapy blooms in the last several years due to the great success in immune checkpoint inhibitor treatment for multiple malignancies (7). Neoantigen-based immunotherapy raising great interests lies in the proof-of-concept investigations on the existence of somatic mutations in malignant cells based on nextgeneration sequencing data. Whole exome sequencing (WES) oriented data mining of mutated peptides not only drafts the mutational landscapes of tumor cells $(8,9)$, but also provides new tools both for the prediction of patient survival (10) and the selection of the peptides as candidate vaccines against tumors in the mice and human (11).

Owing to the implementation of the Cancer Genome Atlas project (TCGA) and the advance in bioinformatics pipeline, BC has been estimated with a mutation load above 10 somatic mutations per megabase of coding DNA comparable to lung cancer and colorectal cancer (12). Considering the availability of $\mathrm{T}$ cell recognition for neoantignes in melanoma with a similar mutation load $(13,14)$, somatic mutation profiles and neoantigen repertoires in BC is worthy of in-depth investigation. What is more, whether somatic mutated peptides can trigger peptide-specific $\mathrm{T}$ cell reactivity in $\mathrm{BC}$ patients is more significant. This might help to decipher the relationship between somatic mutations and clinical outcomes as well as the development of vaccine-based immunotherapy.

In the present study, based on the WES data from 412 BCs in the TCGA database we intended to identify the existence of mutant peptides with neoantigen property in BC. The immunoreactivity to candidate neoantigens was evaluated in BC patients. Correlations between the immunoreactivity to candidate neoantigens and clinical manifestations were further analyzed. This will facilitate the applications of neoantigen-based immunotherapy in BC.

\section{MATERIALS AND METHODS}

\section{Patients and Blood Collection}

One hundred forty $\mathrm{BC}$ patients receiving the surgery in the Department of Urology, Xinhua Hospital affiliated to Shanghai Jiaotong University School of Medicine between Sep 2017 and June 2019 were recruited in the study. The inclusion criteria were: (1) No immunosuppressant in use; (2) histologically confirmed urothelial carcinoma; (3) HLA-A2 ${ }^{+}$. The exclusion criteria were: (1) BC patients receiving preoperative chemotherapy/radiotherapy; (2) BC patients had hematological or autoimmune diseases; (3) Lack of detailed clinical information and laboratory information. Accordingly, 100 patients were excluded. Among them, 82 patients were HLA-A2 negative. Four patients had hematological or autoimmune diseases. Six patients had incomplete medical data and 8 patients had incomplete laboratory data. Finally, a total of 40 patients were enrolled in the study. The clinical-pathological characteristics of 40 HLA-A $2^{+}$BC patients were summarized in Table 1. This study was approved by the Medical Ethics Committee of Xinhua Hospital affiliated to Shanghai Jiaotong University School of Medicine. All the patients enrolled have signed informed consent forms. All the procedures were conducted in accordance with the 
TABLE 1 | Demographic descriptions.

\begin{tabular}{lc}
\hline Characteristics & Cohort (n=40) \\
\hline Mean age \pm SD (years) & $68 \pm 13$ \\
Gender & \\
male & $33(82.5 \%)$ \\
female & $7(17.5 \%)$ \\
Pathology & \\
Pathologic Tumor Stage & \\
Ta, T1 & $32(80 \%)$ \\
T2 & $4(10 \%)$ \\
T3 & $3(7.5 \%)$ \\
T4 & $1(2.5 \%)$ \\
Pathologic Nodal Stage & \\
No & $40(100 \%)$ \\
Histology & \\
Urothelial carcinoma & $40(100 \%)$ \\
Inflammatory Index & \\
Median leukocyte, 10^9/L(IQR) & $6.35(4.93-8.73)$ \\
Median neutrophils, 10^9/L(IQR) & $3.77(2.83-6.39)$ \\
Median neutrophils proportion, \%(IQR) & $61.7(55.65-73.35)$ \\
Mean \pm SD lymphocytes, 10^9/L & $1.59 \pm 0.54$ \\
Mean \pm SD lymphocytes proportion, \% & $24.91 \pm 11.03$ \\
Median monocytes, 10^9/L(IQR) & $0.50(0.40-0.70)$ \\
Mean \pm SD monocytes proportion, \% & $7.95 \pm 2.39$ \\
Median platelets, 10^9/L(IQR) & $180.50(153.25-225.25)$ \\
Median neutrophi//ymphocyte ratio (IQR) & $2.39(1.67-4.99)$ \\
Median platelet/lymphocyte ratio (IQR) & $114.27(89.76-184.72)$ \\
Mean \pm SD lymphocyte/monocyte ratio & $3.27 \pm 1.56$ \\
\hline
\end{tabular}

$I Q R$, interquartile range.

Declaration of Helsinki. Before the surgery, $20 \mathrm{~mL}$ whole blood was collected in EDTA-containing tubes for further experiments.

\section{Data Source}

The WES data of $412 \mathrm{BC}$ tumor and paired peripheral neutrophils were downloaded from the Broad Institute Genome Data Analysis Center (https://gdac.broadinstitute.org/). Protein sequences of transcripts were downloaded from the National Center for Biotechnology Information (NCBI) (https://www.ncbi.nlm.nih. gov/).

\section{Bioinformatics Workflow for Wild Type and Mutant Peptide Prediction}

By mapping to reference sequences in NCBI, a total of 84719 non-synonymous somatic mutations were obtained in 412 BCs. Their transcripts were converted to protein sequences. The sliding window of 9 amino acids was used to generate all possible mutant (MT) peptides covering mutation positions. Corresponding peptides were generated from blood data as wild-type (WT) counterparts. All MT and WT peptides were subjected to the affinity prediction to HLA-A ${ }^{\star} 02: 01$ by using online NetMHCpan software (http://www.cbs.dtu.dk/services/ NetMHCpan-3.0/). MT peptides with strong binding $($ affinity<500) and their WT counterparts with weak binding (affinity $>500$ ) were ranked (Table 2, list 1). Meanwhile, their relative expression levels were ranked by RNAseq data (Table 2, list 2). $\mathrm{R}$ package of RankAggreg was used to aggregate the two rank lists into the final score. The top ranked peptides with high scores were those with strong binding affinity to HLA-A*02:01 and high RNA expression level while WT counterparts had weak binding to HLA-A ${ }^{\star}$ 02:01. The top 57 peptides were synthesized in Shanghai Sangon Biotech (Shanghai, China) and purified through high performance liquid chromatography by the company. They were hydrophilic and were dissolved in phosphate buffer saline at the concentration of $8 \mathrm{mg} / \mathrm{mL}$ for the storage at $-80^{\circ} \mathrm{C}$.

\section{In Vitro Binding Affinity Assay}

T2 cells were maintained in RPMI-IMDM medium containing $10 \%$ fetal bovine serum (FBS) (GIBCO, Grand Island, NY, USA) routinely in the lab. T2 cell-based in vitro binding affinity assay was performed as reported previously (15). Briefly, T2 cells $(0.5 \mathrm{x}$ $\left.10^{6} / \mathrm{mL}\right)$ was incubated with $\beta 2$ microglobulin $(3 \mu \mathrm{g} / \mathrm{mL})$ (Sigma-Aldrich, St. Louis, MO,USA) and individual peptide $(10 \mu \mathrm{g} / \mathrm{mL})$ for $4 \mathrm{~h}$ at $37^{\circ} \mathrm{C}$. L235 peptide was used as a positive control while RPMI-IMDM medium (GIBCO) as a negative control (15). After the incubation, flow cytometric analysis was performed to determine the expression of HLAA2 on T2 cells. Briefly, cells were incubated with PE-mouse anti human-HLA-A2 antibody (Clone BB7.2) (Sigma-Aldrich) for 40 min at $4^{\circ} \mathrm{C}$ and acquired by FACS Canto II (BD Biosciences, Franklin Lakes, NJ, USA). Data analysis was performed by using FlowJo software (Tree Star Inc., Ashland, OR, USA). Mean fluorescence index (MFI) was used to determine the expression level of HLA-A2 molecules on T2 cells. To determine the dissociation constant $(\mathrm{Kd})$ value of each peptide, T2 cells were incubated with $\beta 2$ microglobulin $(3 \mu \mathrm{g} / \mathrm{mL})$ and the peptides at different concentrations $(0,0.4,2,10,20 \mu \mathrm{g} / \mathrm{mL})$ followed by flow cytometric assay. Peptide concentration at $50 \%$ of maximum MFI represented the Kd values of each peptide.

\section{Enzyme-Linked ImmunoSpot (ELISPOT) Assay}

Peptide-specific IFN $\gamma$ release was determined by using an ELISPOT assay according to the manufacturer's instruction (U-CyTech, Utrecht, Netherlands). Briefly, peripheral blood mononuclear cells (PBMCs) were isolated from freshly collected whole blood of BC patients by density gradient centrifugation using LymphoprepTM reagent (Axis-shield, Oslo, Norway). Ninety-six-well PVDF plates (Millipore) were coated with anti-human or mouse IFN- $\gamma$ coating antibody overnight at $4^{\circ} \mathrm{C}$. PBMCs $\left(0.25 \times 10^{6}\right.$ cells/well $)$ were added in the plate and stimulated with individual peptide $(2 \mu \mathrm{g} / \mathrm{mL})$ in RPMI-1640 culture medium containing 10\% FBS, $100 \mathrm{U} / \mathrm{mL}$ penicillin, and $100 \mu \mathrm{g} / \mathrm{mL}$ streptomycin (all from GIBCO) for $20 \mathrm{~h}$ at $37^{\circ} \mathrm{C}$. The secreting IFN- $\gamma$ was detected by biotin-labeled detection antibody and horseradish peroxidase (HRP) conjugated streptavidin. Coloration was developed with AEC substrate solution for $30 \mathrm{~min}$ at room temperature in the dark. The reaction was stopped by thoroughly rinsing the PVDF membrane with demineralized water. The plates were air-dried and the spots were counted by an ELISPOT reader (BioReader Model 4000; Bio-Sys GmbH, Karben, Germany). The number of peptide-specific IFN- $\gamma$ producing cells was calculated as 
TABLE 2 | The information of 57 predicted HLA-A*02:01 restricted wildtype (WT) and mutant (MT) peptide pairs.

\begin{tabular}{|c|c|c|c|c|c|c|c|c|c|c|c|c|c|c|c|}
\hline \multirow[t]{2}{*}{ Gene } & \multirow{2}{*}{$\begin{array}{l}\text { Substitution } \\
\text { (WT,AA\#,MT) }\end{array}$} & \multicolumn{4}{|c|}{ Predicted peptides } & \multirow[t]{2}{*}{ Expression } & \multirow[t]{2}{*}{ Score } & \multirow[t]{2}{*}{ Gene } & \multirow{2}{*}{$\begin{array}{l}\text { Substitution } \\
\text { (WT,AA\#,MT) }\end{array}$} & \multicolumn{4}{|c|}{ Predicted peptides } & \multirow[t]{2}{*}{ Expression } & \multirow[t]{2}{*}{ Score } \\
\hline & & WT & Affinity & MT & Affinity & & & & & WT & Affinity & MT & Affinity & & \\
\hline AHNAK & D4855Y & DLDLKGPKV & 10911.65 & YLDLKGPKKV & 21.03 & 19116.15 & 208186680.1 & EML4 & G786V & QVFGWWPEG & 19946.11 & QVFGWWPEV & 31.08 & 1711.52 & 34084938.73 \\
\hline PPIB & D56Y & DVGRVIFGL & 13532.17 & YVGRVIFGL & 41.73 & 13270.20 & 179020773.9 & DPP9 & R854L & IRAGKPYQL & 18819 & ILAGKPYQL & 21.79 & 1810.09 & 34024615.98 \\
\hline ATF4 & D336Y & IEEVRKA & 17143.93 & YLIEEVRKA & & & 116440272.2 & CC2D1A & P813L & VPAAVPTQV & 20362.16 & VLAAVPTQV & 17.41 & & 32890601.35 \\
\hline MYH9 & E1347K & EMIQLQEEL & 4348.57 & KMIQLQEEL & 46.01 & 25557.15 & 109961178.7 & RUNX1 & R130I & ARFNDLRFV & 16628.02 & AIFNDLRFV & 20.36 & 1926.20 & 31989630.5 \\
\hline CAP1 & K37Q & KAGAAPYV & 11105.08 & SQAGAAPYV & 13.72 & 8707.71 & 96580330.6 & FNDC3B & S1042L & FSETYTFST & 13947.8 & FLETYTFST & 26.58 & 2285.61 & 31818437.06 \\
\hline SYNPO2 & P652L & QPAPWSQPA & 26326.41 & QLAPWSQPA & 40.51 & 3512.41 & 92326931.54 & TP63 & S189L & KSATWTYST & 9726.04 & KLATWTYST & 15.67 & 3218.87 & 31256418.99 \\
\hline RHOB & P75L & YPDTDVILM & 16245.84 & YLDTDVILM & 1503 & 5673.98 & 92092093.75 & LIG1 & P283L & PGQKVPYL & 22825.32 & KLGQKVPYL & & 1317.29 & 30038344.31 \\
\hline & $\mathrm{R} 466 \mathrm{~L}$ & ATVIR & 20843.64 & GLATVI & 29 . & 4335 & & & & 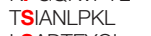 & 9377.99 & TLIANLPKL & 22 & & 29119655.22 \\
\hline KN1A & G61V & 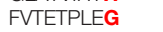 & 14683.37 & ETTETE & ( & 5955 & 307 & & S3S & LSADTEYQI & 6121 & LLADTE & 12 & 4560.5 & 278621 \\
\hline TB & R1 & MKILT & 674.92 & YLMKILT & 18.33 & 12291 & 70664 & ALDH16A1 & G343V & GLDGAVDMG & 18989.79 & GLDGAVDMV & 41.31 & 1376.70 & 26086296.66 \\
\hline PPP2R1A & S314L & ADCRENV & 9585.42 & LLADCRENV & 19.49 & 8184.12 & 78288742.91 & DSG3 & S273L & YSARIEENI & 9423.05 & YLARIEENI & 44.34 & 2684.78 & 25179791.6 \\
\hline ITGB4 & S344L & LDSKVPS & 5661.01 & HLLDSKVPL & 40.66 & 12521.32 & 70374208.67 & MET & R1148Q & LRSEGSPLV & 12216.93 & LQSEGSPLV & 13.19 & 2054.12 & 25067898.47 \\
\hline MKI67 & $\mathrm{H} 84 \mathrm{~L}$ & KHGDVITII & 23696.01 & KLGDVITII & 27.55 & 2799.86 & 66268274.96 & ATP13A1 & R1079L & YLYREAQAR & 11916.14 & YLYREAQAL & 15.58 & 2105.82 & 25060418.29 \\
\hline PRDX1 & F42L & FFFYPLDFT & 5854.1 & FLFYPLDFT & 17.61 & 10921.26946 & 63741879.99 & CD81 & P53S & KPAPNTFYV & 2417.19 & KSAPNTFYV & 48.97 & 10442.95 & 24731207.5 \\
\hline DDB1 & $\mathrm{S} 25 \mathrm{~L}$ & TSAEDLNLL & 10431.97 & TLAEDLNLL & 14.88 & 6098.820878 & 63531965.98 & $\mathrm{PIK} 3 \mathrm{C} 2 \mathrm{~B}$ & $\mathrm{R} 843 \mathrm{Q}$ & KRYYCHSEV & 15638.41 & KQYYCHSEV & 45.87 & 1584.98 & 24713905.56 \\
\hline AZIN1 & G376V & CLLPELNVG & 12266.33 & CLLPELNVV & 29.87 & 3945.14276 & 48274581.58 & TAOK3 & R676L & $\begin{array}{l}\text { IRLQHQTEL } \\
\text { I }\end{array}$ & 26267.8 & ILLQHQTEL & 39. & 930.91 & 24416274.22 \\
\hline TP53 & R273L & FRLGFLHSG & 26951.83 & FLLGFLHSG & 35.3 & $1698.21 \varepsilon$ & 45710122 & & S 85 & FSVDSPDSL & 4088.4 & FLVDSPDSL & 10 & 5673.98 & 23139666.2 \\
\hline CK- & & & & & & & & & & BPEERE & 8412 & RIPERFF & 11 & & 23120 \\
\hline RUVBL1 & P412L & TPANLLAKI & 26279.17 & TLANLL & 4 & 1612.110236 & 55 & $\mathrm{PF}$ & R108W & & 4439.42 & IIDDRNWEL & 34 & 5202.54 & 22916095.26 \\
\hline IRF2BP2 & S195L & GSATPLPTA & 11596.13 & GLATPLPTA & 45.06 & 3548.308467 & 40986759.48 & LRRC14 & P27L & LPRELFPLL & 24049.1 & LLRELFPLL & & 941.28 & 22590297.36 \\
\hline CDC42 & & YPQTDVFLV & 5996.74 & YLQTDVFLV & 2.89 & 6753.099448 & 40477065.13 & MFSD5 & $\mathrm{R} 280 \mathrm{Q}$ & ARAAFWNHV & 18110.07 & AQAAFWNHV & 43.27 & 1246.80 & 22525601.83 \\
\hline HK2 & E774V & LLFRGRISE & 16022.92 & LLFRGRISV & 24.04 & 2502.109804 & 40030954.5 & & F529L & LFSTAEAAV & 9737.94 & LLSTAEAAV & 35.72 & 2306.14 & 22374682.88 \\
\hline CREBBP & R1446L & AYYH & 22971.74 & LLTAVYHEI & 26.32 & 1721.651278 & 38 & MY & D15 & DLTEYRQVL & 15279.98 & YLTEYRQVL & 25.45 & 1446.60 & 22067162.35 \\
\hline SUMO1 & P58L & VPMNSLRFL & 18628.77 & VLMNSLRFL & 19.41 & 2091.24 & 38916550.19 & DNAJC13 & F1325L & LFSKESPLL & 16080.23 & LLSKESPLL & 42.47 & 1366.18 & 21910499.3 \\
\hline TNKS2 & P804L & LPSCYKPQV & 25549.37 & LLSCYKPQV & 19.97 & 1498.065 & 38244701.73 & PCBP1 & S223L & YSIQGQHTI & 2607.71 & YLIQGQHTI & 8.79 & 8300.84 & 21573226.87 \\
\hline EIF4G2 & F855L & LLRFFVHF & 2067.69 & MLLRFFV & 16.6 & 18414.53 & 37768 & NUP210 & $\mathrm{S} 19$ & SSANSILHI & 10602.55 & SLANSILHI & 39 & 2034.74 & 21492140.04 \\
\hline RUNX1 & R205 & RLSELEQ & 19337.71 & RLSEL & & & & & & & 9240.21 & VLDEGGSPI & 42 & 2306.14 & 21212157.43 \\
\hline CDK16 & s9 & AS & & AL & & & 34269380.71 & PNPLA2 & $\mathrm{F} 147 \mathrm{~L}$ & GFIPVYCGL & 8704.87 & GLIPVYCGL & 20.63 & 2399.23 & 20835460.71 \\
\hline $\mathrm{OGDH}$ & D612Y & DILTHIGNV & 11605.41 & YILTHIGNV & 21.58 & 2957.79 & 34262533.63 & & & & & & & & \\
\hline
\end{tabular}

*red colored letters: amino acid substitution. 
spot-forming units (SFUs) per $2.5 \times 10^{5}$ cells of individual peptide minus that of blank control.

\section{Statistical Analysis}

Statistical analysis was performed using SPSS 21 software (IBM SPSS Software, USA). The correlations between peptide-specific SFUs and clinicopathological parameters were analyzed by using Pearson correlation analysis and Student $t$ test. The disease free survival (DFS) originated from the TCGA 412 bladder cancer database and the DFS curves were plotted in silicon based on the cBio Cancer Genomics Portal (http://www.cbioportal.org) that was developed at Memorial Sloan-Kettering Cancer Center (MSKCC) (16-18). The dissociation constant (Kd) value was calculated by Graphpad Prism version 6.0 (Graphpad software Inc., $C A, U S A) . P<0.05$ was considered as statistically significant.

\section{RESULTS}

\section{Prediction of HLA-A*02:01 Restricted WT and MT Peptides From TCGA Whole Exsome Sequencing Data of Bladder Cancer}

Eighty-four thousand seven hundred nineteen mutations from 412 BC patients were downloaded from Broad Institute Genome Data Analysis Center, among which 64,134 mutations were nonsynonymous corresponding to13,718 mutated protein sequences.
They were subjected to online HLA mapping to 34 HLA alleles prevalent in Chinese population. Eight thousand eight hundred twenty-seven peptides restricted to 34 HLA alleles were predicted. Among them, 920 peptides restricted to HLA$A^{\star}$ 02:01 (rank list 1) were selected to analyze their expression levels using RNA-seq data (rank list 2). The final scores of 920 peptides were calculated and the top 57 pairs of WT and MT peptides with HLA-A*02:01 restriction were selected for peptides synthesis. The information of peptide pairs selected, including gene name, mutation site, amino acid sequence, predicted affinity score, gene expression score and the final ranking score, was listed in Table 2. All gene mutation types were missense mutation.

\section{Determination of Binding Affinity to HLA-A2 Molecule}

The top 57 paired HLA-A ${ }^{\star}$ 02:01-restricted MT and WT peptides were synthesized and subjected to an in vitro binding affinity assay using T2 cell. Results from the first set experiment showed that the mean fluorescence indexes (MFIs) of MT peptides were higher than or comparable to those of WT peptides, which is largely consistent with bioinformatics prediction (Figure 1A). We further selected 18 paired peptides whose value of the MFI was 1.5-fold higher in MT peptide than that in WT counterpart for further validation. Higher affinity to HLA-A2 molecules on T2 cells was confirmed in 12 MT peptides when compared to WT peptides (Figure 1B).
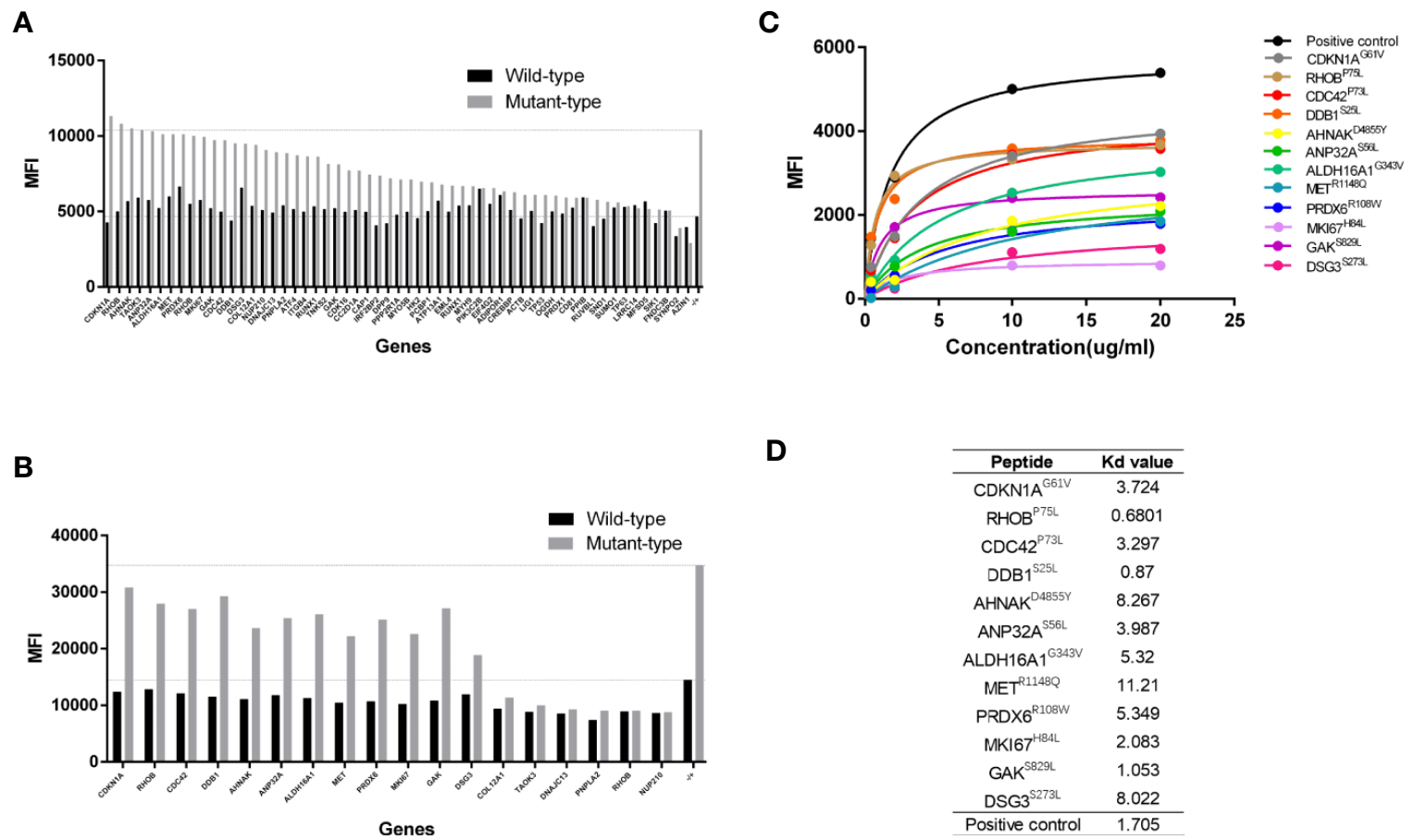

D

\begin{tabular}{cc}
\hline Peptide & Kd value \\
\hline CDKN1A $^{\text {G61V }}$ & 3.724 \\
RHOB $^{\text {P75L }}$ & 0.6801 \\
CDC42 $^{\text {P73L }}$ & 3.297 \\
DDB1 $^{\text {S25L }}$ & 0.87 \\
AHNAK $^{\text {D4855Y }}$ & 8.267 \\
ANP32A $^{\text {S56L }}$ & 3.987 \\
ALDH16A1 $^{\text {G343V }}$ & 5.32 \\
MET $^{\text {R1148Q }}$ & 11.21 \\
PRDX6 $^{\text {R108W }}$ & 5.349 \\
MKI67 $^{\text {H84L }}$ & 2.083 \\
GAK $^{\text {S829L }}$ & 1.053 \\
DSG3 & \\
\hline PositiL & 8.022 \\
\hline
\end{tabular}

FIGURE 1 | Determination of peptide binding affinity to HLA-A2. (A) The mean fluorescence index (MFI) values of HLA-A2 molecules on T2 cells after incubating with 57 pairs HLA-A*0201 restricted wildtype (WT) and mutant (MT) peptides individually. (B) The MFI values of HLA-A2 molecules from 18 pairs HLA-A*0201 WT and MT peptides in a repetitive experiment. (C) The MFI values of HLA-A2 molecules at different concentrations of 12 MT peptides. (D) The Kd values of 12 MT peptides. 
The Kd value of each peptide was further determined by using a series of peptide concentrations when incubating with T2 cells. It was obvious that the MFI values of MT peptides increased along with the elevated peptide concentrations and reached the plateau at $20 \mu \mathrm{g} / \mathrm{mL}$ (Figure 1C) whereas the MFIs of WT peptides did not increased dramatically (Supplementary Figure 1). The Kd values of MT peptides were within the range of 0.6-11 $\mu \mathrm{g} / \mathrm{mL}$ with the highest affinity at $0.6801 \mu \mathrm{g} / \mathrm{mL}\left(\mathrm{RHOB}^{\mathrm{P} 75 \mathrm{~L}}\right)$ and the lowest at $11.21 \mu \mathrm{g} / \mathrm{mL}\left(\mathrm{MET}^{\mathrm{R} 1148 \mathrm{Q}}\right)$ (Figure 1D). However, there was no correlation between the Kd value and the predicted affinity of MT peptides (data not shown).

\section{Determination of the Immunoreactivity to WT and MT Peptides in the Periphery of BC Patients}

Forty HLA-A2 ${ }^{+}$BC patients (Table 1) were recruited to determine the immunoreactivity to 12 paired WT and MT peptides in the periphery by an ELISPOT assay (Figure 2A). PBMCs from each patient were stimulated with $12 \mathrm{WT}$ and MT peptides individually and peptide-specific IFN $\gamma$ SFUs were counted (Figure 2B and Supplementary Table 1). The average SFUs upon in vivo stimulation of $12 \mathrm{WT}$ and MT peptides were compared in 40 BC patients (Figure 2C). It was shown that MT
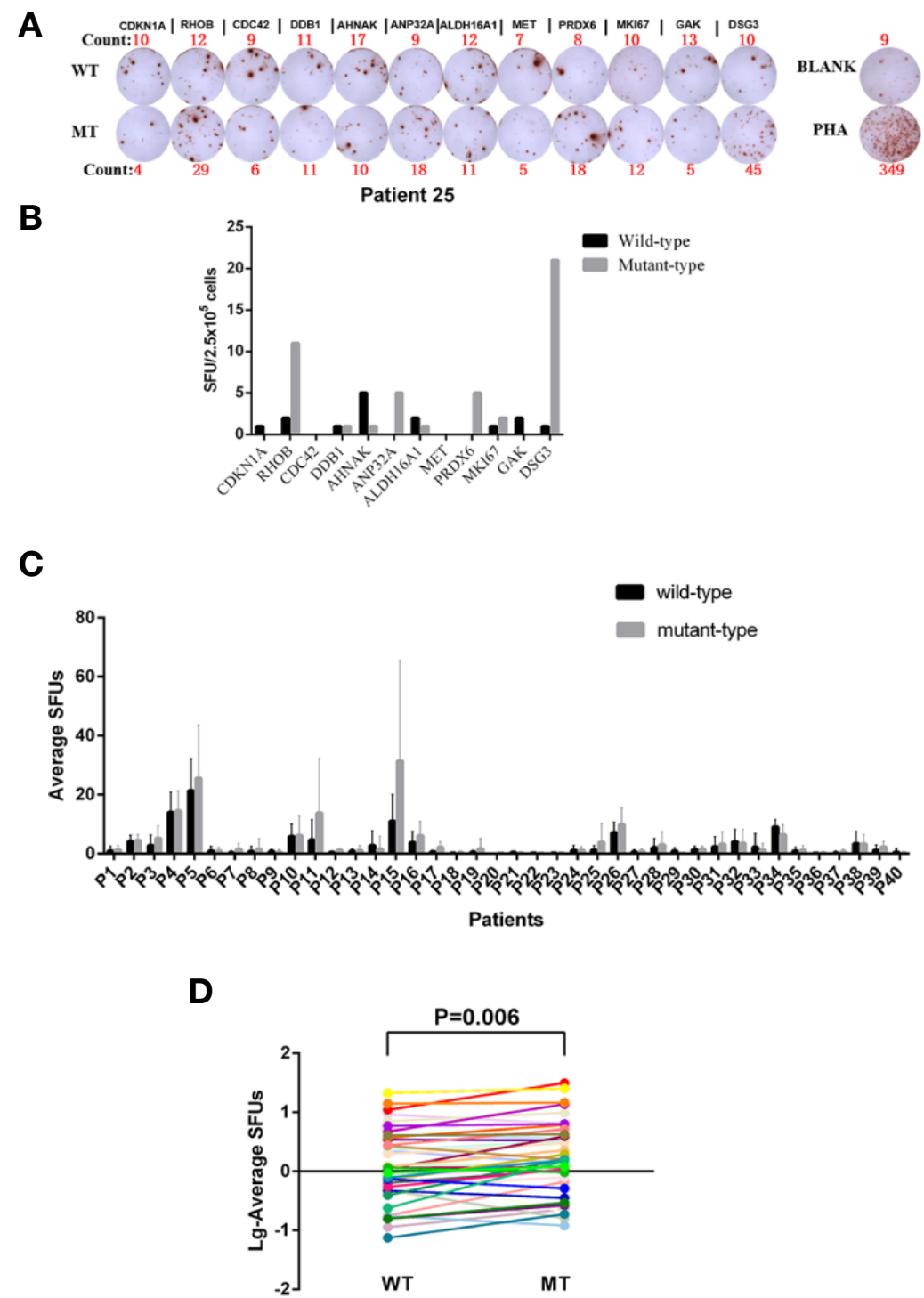

FIGURE 2 | Determination of the Immunoreactivity to WT and MT peptides in HLA-A2 ${ }^{+}$BC patients. (A) Representatives of peptide-specific IFN- $\gamma$ releases by an ELISPOT assay. PBMCs from Patient 25 were isolated and stimulated with $12 \mathrm{WT}$ and MT peptides individually in vitro for $20 \mathrm{~h}$. RPMl $1640 \mathrm{medium}$ was used as BLANK while PHA as a positive control. The number of the spot-forming units (SFUs) of each well was shown as well. (B) The SFUs specific to 12 WT and MT peptides in Patient 25. (C) The average SFUs of 12 WT and MT peptides in $40 \mathrm{HLA}-\mathrm{A} 2^{+} \mathrm{BC}$ patients (the average SFUs $=$ total SFUs from the tested peptides/the number of tested peptides). (D) Comparison of average SFUs between WT and MT peptides by the paired Student $t$ test. Each line represented one patient. 
peptides displayed higher immunoreactivity than WT peptides with elevated average SFUs in $40 \mathrm{BC}$ patients $(\mathrm{P}=0.006)$ (Figure 2D). These results indicate that consistent with high affinity to HLA-A2, MT peptides induce stronger immunoreactivity than WT in the periphery of BC patients. Nevertheless, there is no correlation between the average SFUs of $12 \mathrm{MT}$ peptides and the relevant $\mathrm{Kd}$ value either (data not shown, $\mathrm{P}=0.399$ ). One healthy donor has been added to the screening just for comparison which exhibited few immune-reactivity to peptide stimulation in vitro (Supplementary Figure 2). Considering that stimulating the PBMCs from the patients only for $20 \mathrm{~h}$ maybe not the best condition, we have preformed the ELISPOT assays by stimulation of WT and MT peptides for $20 \mathrm{~h}$ and $60 \mathrm{~h}$, respectively. The SFUs were similar between two time points (Supplementary Figure 3).

\section{Correlations Between MT Peptides- Specific Immunoreactivity and Clinical Manifestations}

With an apparent increase in the immunoreactivity to MT peptides in BC patients, we further performed the correlation analysis between clinical laboratory indications and the average IFN $\gamma$ SFUs specific to MT peptides (MT-SFUs) in 40 BC patients. It was found that there were negative correlations between the average MT-SFUs and the counts of the leukocyte $(r=-0.365$, $\mathrm{P}=0.020$, Figure 3A), the platelet $(\mathrm{r}=-0.455, \mathrm{P}=0.003$, Figure 3B), and thrombocytocrit ( $\mathrm{r}=-0.459, \mathrm{P}=0.004$, Figure 3C). The higher C-reaction protein (CRP) level was, the lower the immunoreactivity to MT peptides displayed ( $\mathrm{P}=0.008$, Figure 3D). The average MT-SFUs was negatively correlated with neutrophil proportion, and positively with monocyte proportion and the count of lymphocytes without statistical significance (Supplementary Figure 4). Subsequently, neutrophil-to-lymphocyte ratio (NLR) (Figure 3E) displayed negative correlation with the average MT-SFUs $(r=-0.326$, $\mathrm{P}=0.040)$ as well as Platelet-to-lymphocyte ratio (PLR) $(\mathrm{r}=-0.387, \mathrm{P}=0.014)$ (Figure 3F).

Considering the variety in the immunoreactivity to MT peptides in $40 \mathrm{BC}$ patients, we subgrouped the patients according to the values of MT-SFUs. Eighteen patients (P2-P5, P10, P11, P15-P17, P25, P26, P28, P31-34, P38, and P39) displayed the immunoreactivity with the SFUs more than 5 upon the stimulation of at least two individual MT peptides, which was defined as HighR group. The resting twenty-two patients (P1, P6-P9, P12-P14, P18-P24, P27, P29, P30, P3537 , and $\mathrm{P} 40$ ) were defined as LowR group (Supplementary Table1). The average SFUs to MT peptides were higher than to WT peptides in HighR group as well $(\mathrm{P}=0.007$, Figure $4 \mathrm{~A})$. Consistently, the numbers of the platelet $(P=0.033$, Figure 4B)
A

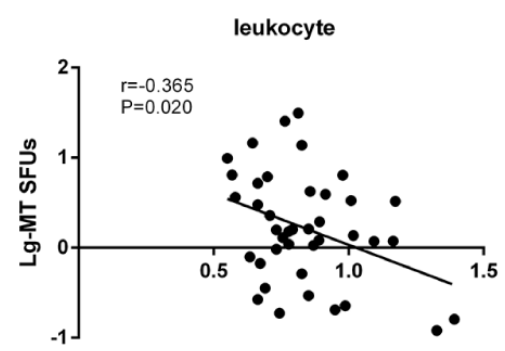

D

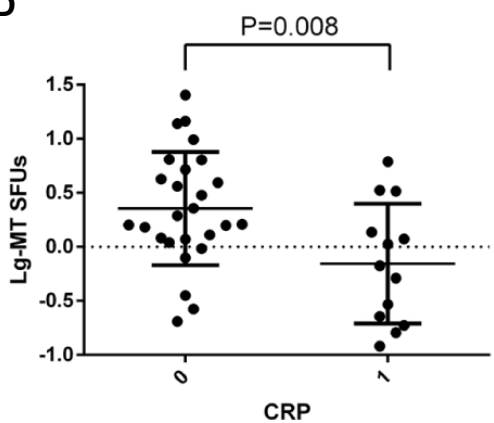

B

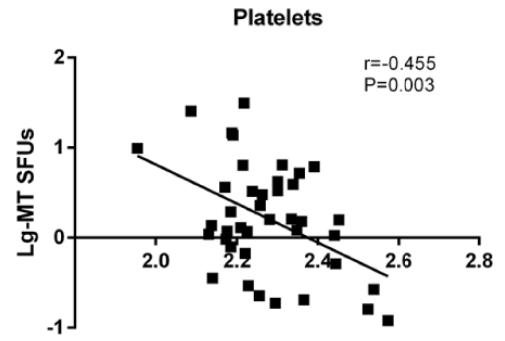

E

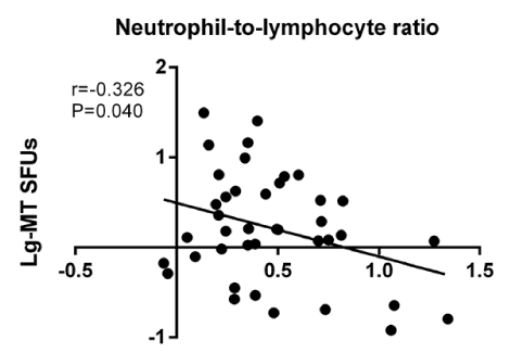

C

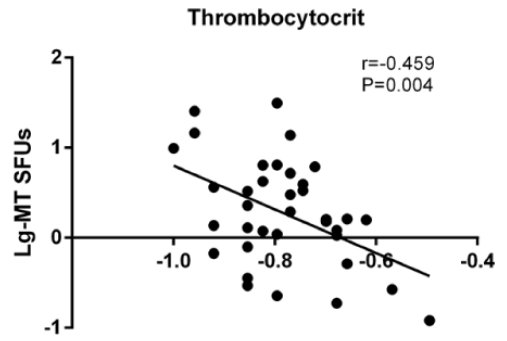

$\mathbf{F}$

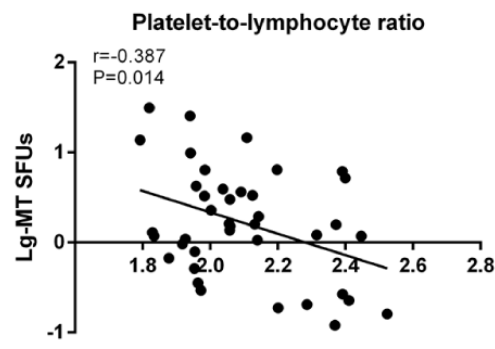

FIGURE 3 | Correlations between MT peptides-specific SFUs and clinical manifestations. (A-C) Correlations between the average MT-specific SFUs (MT-SFUs) and the leukocytes (A), platelets (B) and thrombocytocrit (C). (D) Comparison of the average MT-SFUs between CRP $<8 \mathrm{mg} / \mathrm{mL}$ (indicated as "O") and CRP $\geq 8 \mathrm{mg} / \mathrm{mL}$ (indicated as "1"). (E) Correlation between the average MT-SFUs and neutrophil-to-lymphocyte ratio. (F) Correlation between the average MT-SFUs and platelet-tolymphocyte ratio. Pearson correlation analysis was used for correlation analysis except for CRP by Student $t$ test. 
A

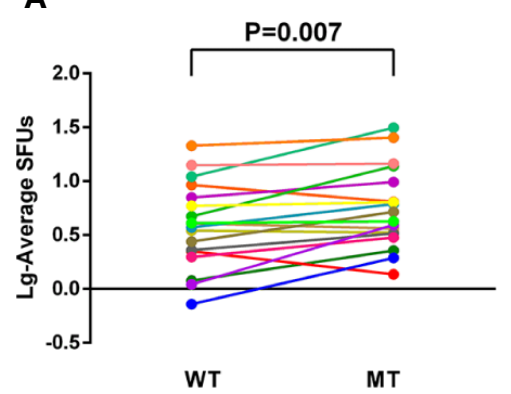

D

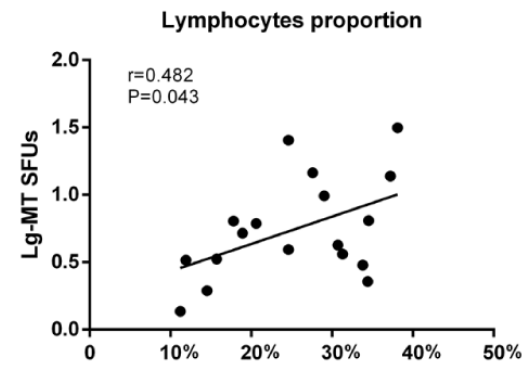

B

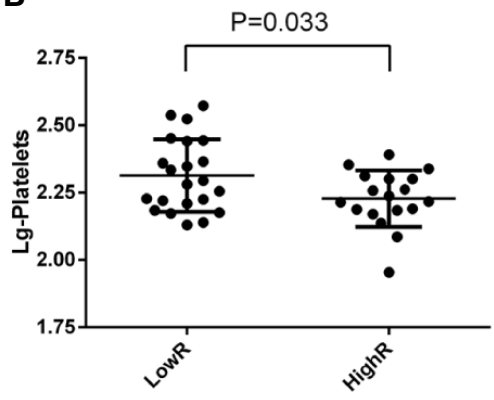

E

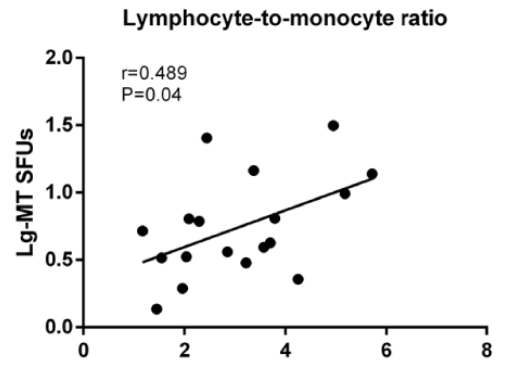

C

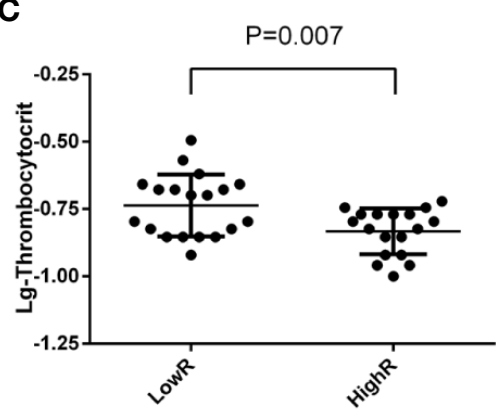

$\mathbf{F}$

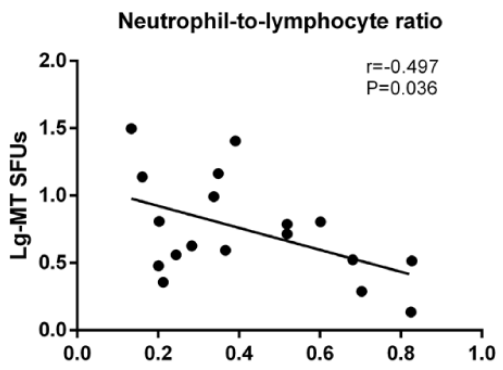

FIGURE 4 | Correlations between MT-SFUs and clinical manifestations in HighR group. Forty HLA-A2 ${ }^{+}$BC patients were subgrouped into LowR ( $\mathrm{n}=22$ ) and HighR ( $n=18$ ) groups according to the SFUs to individual peptides. (A) The SFUs upon WT and MT peptides stimulation were compared in HighR group patients. Each line represented one patient in HighR group. (B, C) Comparison of the platelet (B) and thrombocytocrit (C) between LowR and HighR groups. (D, E) Correlations between the average MT-SFUs in HighR group and lymphocyte proportion (D), LMR(E) and NLR (F). All data were normally distributed after logarithmic transformation and Pearson correlation analysis was used.

and thrombocytocrit $(\mathrm{P}=0.007$, Figure 4C) were lower in HighR group than those in LowR group.

When analyzing the correlations between clinical manifestations and the SFUs to MT peptides in HighR group, it was found that the average MT-SFUs in HighR BC patients exhibited strong positive correlations with the proportion of the lymphocyte $(r=0.482, P=0.043$, Figure $4 D)$ and lymphocyteto-monocyte ratio (LMR, $\mathrm{r}=0.489, \mathrm{P}=0.04$, Figure $4 \mathrm{E}$ ). Negative correlation with NLR $(\mathrm{r}=-0.497, \mathrm{P}=0.036$, Figure 4F) was also present in HighR group (Supplementary Table 2).

\section{Associations Between Hyper- Immunoreactive MT Peptides and Clinical Outcome of BC Patients}

MT peptides are predicted from somatic mutations in tumors, which might in turn facilitate anti-tumor immunity and lead to a better survival. Although the disease free survival (DFS) of $40 \mathrm{BC}$ patients was not reachable due to the short period of the observation, we investigated the relationship between somatic mutations of 12 genes and the DFS by using online cBioportal Website resource including $412 \mathrm{BC}$ patients. The DFS in BC patients with or without somatic mutations were plotted and compared. None of a single gene with somatic mutation dedicated to a significantly longer DFS in $412 \mathrm{BC}$ patients. However, BC patients with CDKN1A, RHOB, DDB1, AHNAK, $A N P 32 A$ and MKI67 genes harboring somatic mutations had relatively longer DFS when compared with unaltered group (Supplementary Figure 5 and Supplementary Table 3).

Considering low frequency of somatic mutations of a single gene in the population, combination of genes with somatic mutations might be more prone in future application. We firstly calculated the positive rate of the immunoreactivity to individual MT peptide in $40 \mathrm{BC}$ patients. Those whose SFUs to a specific MT peptide was more than 5 whereas less than 5 in WT counterpart was defined as positive immunoreactivity. Accordingly, the positive rates of $12 \mathrm{MT}$ peptides in $40 \mathrm{BC}$ patients were calculated. It was found that $\mathrm{CDKN} 1 \mathrm{~A}^{\mathrm{G} 61 \mathrm{~V}}$ and $\mathrm{RHOB}^{\mathrm{P} 75 \mathrm{~L}}$ were the two MT peptides with the highest immunoreactivity-positive rates in $40 \mathrm{BC}$ patients $(22.5 \%$ and $20.0 \%$, respectively) (Figure 5A). We therefore chose six genes including $C D K N 1 A^{G 61 V}$, $R H O B^{P 75 L}, D D B 1^{S 25 L}, A H N A K^{D 4855 Y}, A N P 32 A^{S 56 L}$, and $M K I 67^{H 84 L}$ with high immunoreactivity rates in $40 \mathrm{BC}$ patients and possessed relatively longer DFS for combinational analysis. Their immunoreactivity covered $47.5 \%$ of the patients under investigation. Patients with one or more somatic mutations in 6 
genes exhibited a longer DFS (Median months disease free: 51.41 months) when compared to the cases without mutations (Median months disease free: 29.8 months) although with no significant difference (Log rank Test $P$ value: 0.155 , Figures 5B, C). Therefore, $\mathrm{BC}$ patients harboring 6 hyper-immunoreactive somatic mutations to some extent have a better prognosis in clinic.

\section{DISCUSSION}

Somatic mutations existing in the malignancies can generate neo"nonself" epitopes and is potentiated in inducing an anti-tumor immunity. Previous studies demonstrated the existence of mutation-specific $\mathrm{T}$ cells responses in melanoma $(13,14)$, gastrointestinal cancer and lung cancer $(8,19)$, which facilitates the designing of tumor vaccine as well as peptide-induced adoptive cell therapy in cancer immunotherapy $(20,21)$. In this study, by using mutation data derived from 412 BC WES data in the TCGA database we have identified $12 \mathrm{MT}$ peptides in BC and demonstrated the existence of mutation-specific immunoreactivity in the periphery of $\mathrm{BC}$ patients.

At present the pipeline to define candidate neoantigens from public WES database is well established. In our study, we have filtered MT peptides with both predicted high affinity and expression levels in BC after the comparison to WT counterparts. The top 57 candidate peptide pairs were subjected to the determination of HLA-A2 affinity by a T2 cell-based assay and peptide-specific immunoreactivity in BC patients. Unsurprisingly, the immunoreactivity to MT peptides is dramatically higher than WT peptides. When analyzing the factors associated with mutation- specific immunoreactivity levels in the periphery of $\mathrm{BC}$ patients, the most relevant clinical parameters are the counts of leukocytes, platelets and CRP. Both exhibit significantly negative correlations with mutation-specific immunoreactivity. The leukocytes, platelets and CRP have been already reported to be the indications of systemic inflammation (22). CRP is one of soluble pathogen recognition receptors and increased dramatically after infection (23). The platelets and leukocytes serve as the key initiators and participants of the inflammation (24). During the early stage of the infection, the platelets can adhere to the neutrophils and initiate the formation of neutrophil extracellular traps, which in turn activate the platelets. Once activated, the platelets can secrete multiple cytokines and chemokines to exaggerate inflammation which the leukocytes take in charge. Therefore, the negative correlations between mutation-specific immunity and CRP, the platelets/ leukocytes imply that inflammation might be one of the decay factors of antigen-specific anti-tumor immunity. This is also consistent with the observations and mechanisms on inflammation-related carcinogenesis (25). Interestingly, somatic mutation-specific immunoreactivity was negatively correlated in more extent with NLR, PLR and LMR in BC patients and high immunoreactive group, which is also the indicators of inflammation $(22,26)$. Higher NLR is reported to be associated with a poor prognosis during immune checkpoint inhibitor treatment $(27,28)$, another type of immunotherapy relying on the restore of anti-tumor immunity. These results altogether support that the control of inflammation during cancer immunotherapy is probably benefit for the better outcome of the treatment.

According to our results, the immunoreactivity to somatic mutation-derived peptides is very diverse in BC patients. Fifty-five
A

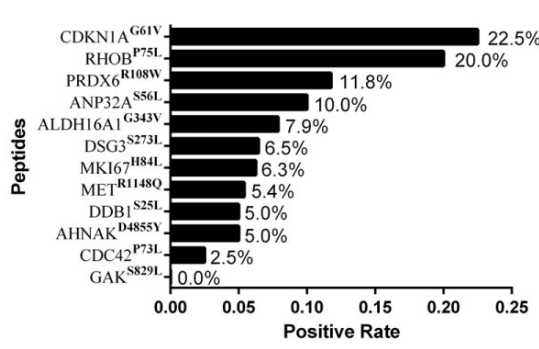

C

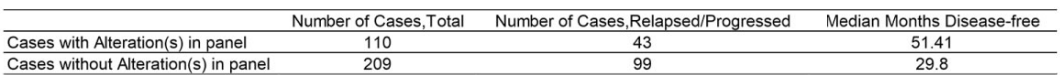

FIGURE 5 | Somatic mutations and clinical outcome from TCGA database. (A) Positive rates of the immunoreactivity to individual MT peptides in 40 HLA-A2 ${ }^{+}$BC patients. (B) Kaplan-Meier curve of BC patients with (altered group) or without mutations (unaltered group) in 6 genes from the TCGA database. (C) The number of the cases and median disease-free months of BC patients with or without mutations in 6 genes. 
percent (22/40) of BC patients exhibited rare mutation-specific SFUs while the remaining patients displayed relatively high responses. Although we did not get the information of gene mutant patterns in BC patients investigated, we have surveyed the mutant rates in 412 $\mathrm{BC}$ samples by the WES data online. The frequency of mutation rates is very low (from 1-6 cases in 412 BC patients). Nevertheless, mutation-specific immunoreactivity is detectable in most of the HighR patients. We therefore deduce that immune recognition of somatic mutations might be more sensitive than next generation sequencing based detection of somatic mutations in tumor patients. In our study, the immunoreactivity to WT peptides is also observed in certain $\mathrm{BC}$ patients with a less extent and one healthy donor have been added to the screening which exhibited few immune-reactivity to peptide stimulation in vitro. A recent publication has described the generation of neoantigen-specific $\mathrm{T}$ cells from healthy donors (29), which in part support our results that WT-specific T cells might be the evidence on TCR redundancy in BC patients. Another possibility might be due to the overexpression of genes in tumors, which also facilitates the induction of immunoreacitivity in cancer patients. Three genes, including MKI67, DDB1, and ANP32A have been reported to be upregulated in BC already (http://ualcan.path. uab.edu/index.html) (30).

Although most of the studies define mutation-specific immunity in tumor microenvironments (TME) using tumor infiltrating lymphocytes (31), the existence of mutation-specific immunoreactivity in the periphery is still significant. According to a recent report, pre-existing $\mathrm{T}$ cells infiltrating in TME were too fragile to be revigorated upon immune checkpoint inhibitor treatment. Peripheral antigen-specific immune cells become the resources that circulate and replace the exhausted $\mathrm{T}$ cells inside TME to function as the anti-tumor players (32). MT peptide-specific $\mathrm{T}$ cells in the periphery can act as the reservoir of anti-tumor immunity once these mutant peptides are applied as tumor vaccine against $\mathrm{BC}$. In addition, MT peptides might also be subjected to the combination therapy with intravesical BCG administration or other immunotherapy in the future. While intravesical BCG administration induces the infiltration of immune cells inside in a less antigen-specific manner, addition of MT peptides with highimmunogenicity recapitulates the in vivo sensitization and orchestrates anti-tumor immunity in an antigen-specific manner.

Taken together, by using public WES data from 412 bladder cancer in the TCGA database we have identified 12 HLA-A $^{\star} 02: 01$ restricted MT peptides exhibiting stronger immunoreactivity in the periphery of $\mathrm{BC}$ patients among which six somatic mutations are partially associated with a better prognosis. MT peptide-specific immunoreactivity is negatively correlated with peripheral inflammatory indicators, providing a potential strategy to enhance the efficacy of neoantigen-based immunotherapy against urothelial carcinoma in the future.

\section{REFERENCES}

1. Siegel RL, Miller KD, Jemal A. Cancer statistics, 2018. CA:Cancer J Clin (2018) 68: (1):7-30. doi: 10.3322/caac.21442

2. Ferlay J, Colombet M, Soerjomataram I, Dyba T, Randi G, Bettio M, et al. Cancer incidence and mortality patterns in Europe: Estimates for 40 countries and 25 major cancers in 2018. Eur J Cancer (2018) 103:356-87. doi: 10.1016/j.ejca.2018.07.005

\section{DATA AVAILABILITY STATEMENT}

The original contributions presented in the study are included in the article/Supplementary Material. Further inquiries can be directed to the corresponding authors.

\section{ETHICS STATEMENT}

The studies involving human participants were reviewed and approved by Medical Ethics Committee of Xinhua Hospital affiliated to Shanghai Jiaotong University School of Medicine. The patients/participants provided their written informed consent to participate in this study.

\section{AUTHOR CONTRIBUTIONS}

YW and HS conceived the project and designed the experiments. CW, YD, and YL performed the experiments. QZ and ZC performed the bioinformatics analysis. CW, YD, SX, and HD collected the blood samples and clinical data. LX, SW, and PJ provided technique support. GZ, YW, and WH initiated the projects. YW, HS, and CW performed data analysis. YW, HS, ZC, CW, YD, and QZ drafted the manuscript. YW and HS were senior authors. All authors contributed to the article and approved the submitted version.

\section{FUNDING}

This work was supported by the grant from National Key R\&D Program of China (2016YEC1303300) and Shanghai Academic Research Leader Project (2018XD1403300).

\section{ACKNOWLEDGMENTS}

We appreciated Dr. Yaozhou Shi and Dr. Yu Ye from Sinotec Genomics Company for their suggestion on peptide prediction from the TCGA database.

\section{SUPPLEMENTARY MATERIAL}

The Supplementary Material for this article can be found online at: https://www.frontiersin.org/articles/10.3389/fimmu.2020. 576603/full\#supplementary-material

3. Chen W, Zheng R, Baade PD, Zhang S, Zeng H, Bray F, et al. Cancer statistics in China, 2015. CA: Cancer J Clin (2016) 66(2):115-32. doi: 10.3322/ caac. 21338

4. Chang SS, Boorjian SA, Chou R, Clark PE, Daneshmand S, Konety BR, et al. Diagnosis and Treatment of Non-Muscle Invasive Bladder Cancer: AUA/ SUO Guideline. J Urol (2016) 196(4):1021-9. doi: 10.1016/j.juro. 2016.06.049 
5. Redelman-Sidi G, Glickman MS, Bochner BH. The mechanism of action of BCG therapy for bladder cancer-a current perspective. Nat Rev Urol (2014) 11(3):153-62. doi: 10.1038/nrurol.2014.15

6. Taniguchi K, Koga S, Nishikido M, Yamashita S, Sakuragi T, Kanetake H, et al. Systemic immune response after intravesical instillation of bacille Calmette-Guérin (BCG) for superficial bladder cancer. Clin Exp Immunol (1999) 115(1):131-5. doi: 10.1046/j.1365-2249.1999.00756.x

7. Couzin-Frankel J. Breakthrough of the year 2013Cancer immunotherapy. Science (2013) 342(6165):1432-3. doi: 10.1126/science.342.6165.1432

8. Parkhurst MR, Robbins PF, Tran E, Prickett TD, Gartner JJ, Jia L, et al. Unique Neoantigens Arise from Somatic Mutations in Patients with Gastrointestinal Cancers. Cancer Discov (2019) 9(8):1022-35. doi: 10.1158/ 2159-8290.CD-18-1494

9. Liu S, Matsuzaki J, Wei L, Tsuji T, Battaglia S, Hu Q, et al. Efficient identification of neoantigen-specific T-cell responses in advanced human ovarian cancer. J Immunother Cancer (2019) 7(1):156. doi: 10.1186/s40425-019-0629-6

10. Kadara H, Choi M, Zhang J, Parra ER, Canales JR, Gaffney SG, et al. Wholeexome sequencing and immune profiling of early-stage lung adenocarcinoma with fully annotated clinical follow-up. Ann Oncol (2017) 28(1):75-82. doi: 10.1093/annonc/mdw436

11. Hundal J, Carreno BM, Petti AA, Linette GP, Griffith OL, Mardis ER, et al. pVAC-Seq: A genome-guided in silico approach to identifying tumor neoantigens. Genome Med (2016) 8(1):11. doi: 10.1186/s13073-016-0264-5

12. Schumacher TN, Schreiber RD. Neoantigens in cancer immunotherapy. Science (2015) 348(6230):69-74. doi: 10.1126/science.aaa4971

13. Ott PA, Hu Z, Keskin DB, Shukla SA, Sun J, Bozym DJ, et al. An immunogenic personal neoantigen vaccine for patients with melanoma. Nature (2017) 547 (7662):217-21. doi: 10.1038/nature22991

14. Sahin U, Derhovanessian E, Miller M, Kloke BP, Simon P, Löwer M, et al. Personalized RNA mutanome vaccines mobilize poly-specific therapeutic immunity against cancer. Nature (2017) 547(7662):222-6. doi: 10.1038/ nature 23003

15. Jin S, Wang Y, Zhang Y, Zhang HZ, Wang SJ, Tang JQ, et al. Humoral immune responses against tumor-associated antigen OVA66 originally defined by serological analysis of recombinant cDNA expression libraries and its potentiality in cellular immunity. Cancer Sci (2008) 99(8):1670-8. doi: $10.1111 / j .1349-7006.2008 .00860 . x$

16. Cerami E, Gao J, Dogrusoz U, Gross BE, Sumer SO, Aksoy BA, et al. The cBio cancer genomics portal: an open platform for exploring multidimensional cancer genomics data. Cancer Discov (2012) 2(5):401-4. doi: 10.1158/21598290.CD-12-0095

17. Gao J, Aksoy BA, Dogrusoz U, Dresdner G, Gross B, Sumer SO, et al. Integrative analysis of complex cancer genomics and clinical profiles using the cBioPortal. Sci Signaling (2013) 6(269):pl1. doi: 10.1126/ scisignal.2004088

18. Robertson AG, Kim J, Al-Ahmadie H, Bellmunt J, Guo G, Cherniack AD, et al. Comprehensive Molecular Characterization of Muscle-Invasive Bladder Cancer. Cell (2017) 171(3):540-56.e25. doi: 10.1016/j.cell.2017.09.007

19. Veatch JR, Jesernig BL, Kargl J, Fitzgibbon M, Lee SM, Baik C, et al. Endogenous CD4(+) T Cells Recognize Neoantigens in Lung Cancer Patients, Including Recurrent Oncogenic KRAS and ERBB2 (Her2) Driver Mutations. Cancer Immunol Res (2019) 7(6):910-22. doi: 10.1158/23266066.CIR-18-0402

20. Li L, Goedegebuure SP, Gillanders WE. Preclinical and clinical development of neoantigen vaccines. Ann Oncol (2017) 28(suppl_12):xii11-xii7. doi: 10.1093/annonc/mdx681
21. Capietto AH, Jhunjhunwala S, Delamarre L. Characterizing neoantigens for personalized cancer immunotherapy. Curr Opin Immunol (2017) 46:58-65. doi: 10.1016/j.coi.2017.04.007

22. Cantiello F, Russo GII, Vartolomei MD, Farhan ARA, Terracciano D, Musi G, et al. Systemic Inflammatory Markers and Oncologic Outcomes in Patients with High-risk Non-muscle-invasive Urothelial Bladder Cancer. Eur Urol Oncol (2018) 1(5):403-10. doi: 10.1016/j.euo.2018.06.006

23. Tanaka N, Kikuchi E, Shirotake S, Kanao K, Matsumoto K, Kobayashi H, et al. The predictive value of $\mathrm{C}$-reactive protein for prognosis in patients with upper tract urothelial carcinoma treated with radical nephroureterectomy: a multi-institutional study. Eur Urol (2014) 65(1):227-34. doi: 10.1016/j.eururo.2012.11.050

24. Herter JM, Rossaint J, Zarbock A. Platelets in inflammation and immunity. Journal of thrombosis and haemostasis. JTH (2014) 12(11):1764-75. doi: $10.1111 /$ jth. 12730

25. Greten FR, Grivennikov SII. Inflammation and Cancer: Triggers, Mechanisms, and Consequences. Immunity (2019) 51(1):27-41. doi: 10.1016/j.immuni.2019.06.025

26. Templeton AJ, Mcnamara MG, Seruga B, Badillo FEV, Aneja P, Ocaña A, et al. Prognostic role of neutrophil-to-lymphocyte ratio in solid tumors: a systematic review and meta-analysis. J Natl Cancer Inst (2014) 106(6):dju124. doi: 10.1093/jnci/dju124

27. De Giorgi U, Procopio G, Giannarelli D, Sabbatini R, Bearz A, Buti S, et al. Association of Systemic Inflammation Index and Body Mass Index with Survival in Patients with Renal Cell Cancer Treated with Nivolumab. Clin Cancer Res (2019) 25(13):3839-46. doi: 10.1158/1078-0432.CCR-18-3661

28. Diem S, Schmid S, Krapf M, Flatz L, Born D, Jochum W, et al. Neutrophil-toLymphocyte ratio (NLR) and Platelet-to-Lymphocyte ratio (PLR) as prognostic markers in patients with non-small cell lung cancer (NSCLC) treated with nivolumab. Lung Cancer (2017) 111:176-81. doi: 10.1016/ j.lungcan.2017.07.024

29. Ali M, Foldvari Z, Giannakopoulou E, Böschen ML, Strønen E, Yang W, et al. Induction of neoantigen-reactive $\mathrm{T}$ cells from healthy donors. Nat Protoc (2019) 14(6):1926-43. doi: 10.1038/s41596-019-0170-6

30. Chandrashekar DS, Bashel B, Balasubramanya SAH, Creighton CJ, Rodriguez IP, Chakravarthi BVSK, et al. UALCAN: A Portal for Facilitating Tumor Subgroup Gene Expression and Survival Analyses. Neoplasia (2017) 19 (8):649-58. doi: 10.1016/j.neo.2017.05.002

31. Pfannstiel C, Strissel PL, Chiappinelli KB, Sikic D, Wach S, Wirtz RM, et al. The Tumor Immune Microenvironment Drives a Prognostic Relevance That Correlates with Bladder Cancer Subtypes. Cancer Immunol Res (2019) 7 (6):923-38. doi: 10.1158/2326-6066.CIR-18-0758

32. Yost KE, Satpathy AT, Wells DK, Qi Y, Wang C, Kageyama R, et al. Clonal replacement of tumor-specific T cells following PD-1 blockade. Nat Med (2019) 25(8):1251-9. doi: 10.1038/s41591-019-0522-3

Conflict of Interest: The authors declare that the research was conducted in the absence of any commercial or financial relationships that could be construed as a potential conflict of interest.

Copyright (๑) 2020 Wang, Ding, Liu, Zhang, Xu, Xia, Duan, Wang, Ji, Huang, Zhao, Cao, Shen and Wang. This is an open-access article distributed under the terms of the Creative Commons Attribution License (CC BY). The use, distribution or reproduction in other forums is permitted, provided the original author(s) and the copyright owner(s) are credited and that the original publication in this journal is cited, in accordance with accepted academic practice. No use, distribution or reproduction is permitted which does not comply with these terms. 